\title{
Contribuições ao Estudo dos Valores Pessoais, Laborais e Organizacionais
}

\author{
Alvaro Tamayo ${ }^{1}$ \\ Universidade de Brasília \\ Universidade Católica de Brasília
}

\begin{abstract}
RESUMO - Este trabalho teve por objetivo fazer um breve histórico do meu interesse pelo estudo dos valores e discutir algumas das minhas contribuições científicas na área dos valores pessoais, laborais e organizacionais. O estímulo para escrever este texto foi o recebimento do título de professor emérito da Universidade de Brasília. Inicialmente, descrevi a minha trajetória de ensino e de pesquisa na Université de Moncton, no Canadá e na Universidade de Brasília. Os elementos discutidos neste relato representam indicadores da minha contribuição ao estudo dos valores pessoais, laborais e organizacionais.
\end{abstract}

Palavras-chave: valores pessoais; valores laborais; valores organizacionais.

\section{Contributions to the Study of Personal, Work, and Organizational Values}

\begin{abstract}
The purpose of this paper is to briefly report my interest for the study of values and discuss some of my scientific contributions in the domain of personal, labor and company values. The encouragement to write this text was the receiving of the emeritus professor title by the Universidade de Brasília. To begin with, I described my teaching and researching experience at Université de Moncton, in Canada and at Universidade de Brasília. The elements discussed in this report represent indicators of my contribution to the study of personal, labor and company values.
\end{abstract}

Key words: personal values; labor values; company values.

Understanding human values is a never-ending process $-a$ groping toward an ultimate objective that can be attained only by a method of successive approximation."

(Rokeach, 1979, p. 71).

O objetivo deste trabalho é relatar a gênese do meu interesse pelo estudo dos valores e de discutir, brevemente, algumas das minhas contribuições na área dos valores pessoais, laborais e organizacionais. O estímulo para escrever este texto foi o recebimento do título de professor emérito da Universidade de Brasília. Acho que uma forma de corresponder e de agradecer à UnB e a Instituto de Psicologia por esta honrosa distinção seria de descrever a minha contribuição como professor e pesquisador nesta instituição. Seria uma tarefa abrangente, difícil de ser colocada em poucas linhas. Portanto, neste texto limitar-me-ei a comentar a minha contribuição científica no contexto da minha linha de pesquisa principal que tem como objetivo o estudo dos valores humanos. Antes de abordar este tema, considero importante descrever, brevemente, a minha trajetória de ensino e de pesquisa. Iniciei o ensino universitário em 1970 na Université de Moncton, no Canadá. Desde o início participei do ensino de graduação e pós-graduação. Durante os oito anos que trabalhei nessa universidade tive a oportunidade de conviver e de pesquisar com colegas formados nas mais diversas universidades canadenses e estadunidenses. Como a minha formação era mais de tipo europeu, o convívio com pessoas formadas em Norte-América foi muito enriquecedor para mim. Em 1978 iniciei as minhas atividades docentes na

1 Endereço: SHIN QI 03 conj. 04 casa 02 Lago Norte, Brasília, DF, Brasil 71500-240.E-mail: tamayo@terra.com.br
Universidade de Brasília. O meu vínculo com esta instituição desenvolveu-se progressivamente. No início, o meu projeto de vinculação com a UnB e de estadia no Brasil não ia além de um ano acadêmico. Fatores de diversa ordem influenciaram a formação de um vínculo mais sólido e permanente. As minhas pesquisas, nas duas primeiras décadas da minha carreira docente, giravam em torno do problema da atribuição. Desde o início de minha formação tive interesse pelo estudo da tendência das pessoas a explicar os seus próprios comportamentos e o comportamento dos outros por meio da atribuição de características estáveis. Desta forma, as minhas primeiras linhas de pesquisa estudavam problemas relacionados com autoconceito, estereótipos, locus de controle e atribuição de causalidade e de responsabilidade. $\mathrm{O}$ meu interesse pelo estudo dos valores surgiu muito mais tarde.

\section{Duas décadas pesquisando valores}

A minha primeira pesquisa na área de valores foi em 1986. Ela foi realizada com o inventário de valores de Rokeach (IVR), adaptado para o Brasil por Günther (1981). O inventário de valores de Rokeach consta de 36 itens distribuídos em duas listas de 18 valores cada, sendo a primeira de valores terminais (exemplo, liberdade, igualdade, honestidade) e a segunda de instrumentais (independente, leal, honesto). Cada valor é definido operacionalmente por meio de uma curta frase. A minha pesquisa tinha como objetivo levantar as prioridades de valores de uma amostra de 1.258 adolescentes, estudantes universitários e adultos de Brasília. Na época eu fazia parte de um grupo de psicólogos sociais que todos os anos apresentava, no congresso de psicologia de Ribeirão Preto, um simpósio intitulado "Quem é o brasileiro". O objetivo deste simpósio 


\section{A. Tamayo}

era de estudar e divulgar características psicossociais do brasileiro. Cada participante do simpósio apresentava resultados de pesquisa empírica sobre um tema de sua escolha relacionado com alguma característica psicossocial do brasileiro. Em 1986, a minha opção foi pelos valores. A apresentação dos resultados desta pesquisa no congresso teve grande sucesso entre os participantes do simpósio devido, sem dúvida, ao fato de que na época não existiam dados sobre os valores do brasileiro, ou melhor, com amostras compostas por sujeitos brasileiros. Possivelmente um segundo fator responsável pelo sucesso dos resultados foi o fato de que, com o inventário de Rokeach, os resultados significativos são numerosos já que este instrumento não apresenta nenhuma estrutura em fatores ou dimensões, de tal forma que cada um dos valores é analisado individualmente.

Apesar do sucesso da pesquisa no congresso, pessoalmente fiquei frustrado e sem vontade de continuar pesquisando na área dos valores. Esta insatisfação foi provocada por três características do instrumento de medida utilizado - o IVR -, e da teoria subjacente. Em primeiro lugar, a representatividade dos valores humanos contida nos itens do IVR é muito baixa. Os critérios utilizados por Rokeach para a construção dos itens foram pouco adequados para atender às exigências de uma amostra representativa dos valores humanos. Os critérios para a construção dos itens terminais e instrumentais não foram os mesmos. Os valores terminais foram identificados a partir da literatura que evocava valores americanos ou de outras sociedades. Além disso, o autor solicitou a um grupo de 30 estudantes de pós-graduação em psicologia que propusessem valores e entrevistou uma amostra de 100 adultos sobre os seus valores. Esta amostra era representativa de uma pequena cidade norte-americana. Desta forma obteve várias centenas de valores. Rokeach eliminou os sinônimos, os valores muito específicos e aqueles que não representavam metas da existência. Os itens representando os valores instrumentais foram construídos a partir de uma lista de 555 palavras relativas a traços de personalidade, compilada por Anderson em 1968. As fontes, portanto, utilizadas para a construção dos itens, tanto terminais quanto instrumentais, são muito limitadas e, em consequiência, estes dificilmente representam uma amostra dos valores humanos. Em segundo lugar, a sua falta de estrutura, isto é, a falta de relação intrínseca entre os valores. Do ponto de vista estatístico e teórico cada valor constitui uma variável independente de todas as outras. Esta situação favorece a multiplicidade de resultados significativos, mas dificulta a explicação dos mesmos. Qual o significado, por exemplo, da diferença em aproximadamente 15 valores observada entre homens e mulheres? Em princípio precisaria uma hipótese explicativa para cada um dos resultados ou tentar fazer, a posteriori, uma espécie de análise fatorial subjetiva para agrupar os valores em conjuntos significativos e, a partir daí, explicar os resultados obtidos. Com grande dificuldade consegui discutir superficialmente, por exemplo, as numerosas e aparentemente díspares diferenças observadas entre adolescentes, estudantes universitários e adultos (Tamayo, 1988). Em terceiro lugar, a escala utilizada no Inventário de valores de Rokeach é de tipo ordinal. Os sujeitos são solicitados de ordenar os valores por ordem de importância. Além do aspecto tedioso e complicado deste tipo de atividade, a distância entre os números não é igual, isto é, os números não são métricos, eles representam somente postos e não quantidades que podem ser adicionadas. Para a realização da minha pesquisa, eu já tinha substituído a escala ordinal por uma escala intervalar que permitia a realização de estatística paramétrica. Os três problemas indicados foram decisivos para que eu considerasse encerrada a minha incursão na área dos valores. Em 1987, Schwartz e Bilsky publicaram um artigo no Journal of Personality and Social Psychology intitulado Toward a universal psychological structure of human values. Eu não tomei conhecimento desta publicação porque, como o meu interesse pelos valores tinha sido passageiro, eu não estava acompanhando a literatura nesta área de forma sistemática.

Em 1987, Shalom Schwartz, professor da Universidade Hebráica de Jerusalém, acabava de lançar um projeto para estudar os valores e, particularmente, para tentar identificar uma possível estrutura universal dos valores. Desta forma, começou a formar uma equipe de pesquisadores em todos os continentes para participar no seu projeto. A finais de 1989 recebi convite para fazer parte da sua equipe. Junto com o convite recebi cópia da publicação de 1987, uma síntese da sua teoria, cópia do instrumento provisório e estratégias para o aprimoramento do mesmo e as linhas metodológicas gerais para a verificação da sua teoria. Ao ler a documentação por ele encaminhada, encontrei resposta aos meus questionamentos relativos à medida e à falta de estrutura e fiquei entusiasmado com a teoria proposta. Aceitei com prazer participar da equipe de pesquisa transcultural e desde então o estudo dos valores transformou-se na minha linha de pesquisa principal.

Apesar de praticamente existir consenso entre os autores de que os valores são representações cognitivas das necessidades, Schwartz foi o primeiro a imaginar uma estrutura dos valores baseada na motivação subjacente a cada um deles. $\mathrm{O}$ que diferencia um valor de outro é o tipo de motivação que ele expressa. Uma das grandes contribuições de Schwartz foi ter identificado as motivações que expressam os valores. Schwartz (2005) verificou empiricamente com amostras de mais de 67 países uma estrutura axiológica composta por 10 motivações. Indícios estatísticos sugerem que os 10 tipos motivacionais identificados cobrem todo o espectro motivacional dos valores. Contudo, o próprio Schwartz (2005, pp. 45-46) afirma que "é difícil rejeitar definitivamente a possibilidade de que alguns tipos motivacionais universais estejam ausentes". Na ciência, a teoria é uma representação simplificada do mundo real, físico ou social. A elaboração de um modelo é progressiva e implica na necessidade de um trajeto continuo de ida-e-volta entre a realidade e o modelo. A estrutura dos valores foi construída nesse movimento incessante entre o fenômeno observado e o modelo proposto. Assim, de oito (Schwartz \& Bilsky, 1987), os tipos motivacionais passaram para 11, vários deles mudaram de nome e, finalmente, se estabilizaram em 10 (Schwartz, 2005). Recentemente, Wach e Hammer (2003) postularam a possível ausência de dois tipos motivacionais relativos à procura da verdade: a motivação que sustenta a busca da verdade racional de um lado, e a verdade irracional ou mística de outro lado. No Brasil já realizamos pesquisa tentando verificar a existência de mais estas duas motivações subjacentes aos valores; atualmente os dados estão sendo analisados. 


\section{Valores pessoais}

O instrumento para avaliação dos valores proposto por Schwartz, o Schwartz Value Survey (SVS), é muito semelhante ao de Rokeach. Está composto por 57 valores, distribuídos em duas listas, que devem ser avaliados numa escala que vai de -1 a 7 . Apesar da sua semelhança com o inventário de Rokeach, ele supera as principais deficiências naquele detectadas: 1) os valores que constituem os seus itens formam uma amostra mais representativa dos valores humanos. Schwartz integrou no seu instrumento valores de outras culturas, tais como chinesa, muçulmana, drusa, etc;2) a escala utilizada é de tipo intervalar e 3) o seu instrumento, SVS, apresenta estrutura de tipo fatorial e avalia os valores de acordo com os tipos motivacionais propostos na teoria. Existe, portanto, uma estrutura de relações dinâmicas entre os valores e entre os tipos motivacionais de valores. Além disso, todos os itens do instrumento e os tipos motivacionais de valores organizam-se ao longo de um continuum. O SVS, porém, ainda apresenta deficiências menores. Primeiramente, ele está dividido em duas listas de itens correspondendo à divisão dos valores estabelecida por Rokeach em valores terminais e instrumentais, os primeiros formulados com substantivos e os segundo com adjetivos. Schwartz (1992), ao utilizar o SVS nos mais diversos países, observou que nas sociedades onde o substantivo e o adjetivo não se diferenciam, a distinção entre valores instrumentais e terminais não era observada. Os resultados obtidos anteriormente por Rokeach e por outros pesquisadores eram conseqüência da forma diferencial como estavam formulados os itens e não de uma característica estrutural dos valores. Desta forma, a presença das duas listas de valores no instrumento atual constitui um anacronismo desnecessário e contra as evidências empíricas. Em segundo lugar, a escala introduzida por Schwartz varia de 0 a 6 , com dois pontos extra-escala: -1 representando que o valor assim avaliado "é oposto aos princípios que orientam sua vida" e 7 cujo significado é que o valor assim avaliado "é de suprema importância como um princípio orientador em sua vida; geralmente, uma pessoa não possui mais de dois desses valores". A utilização de -1 para identificar os valores opostos não tem apresentado nenhuma dificuldade. A avaliação, porém, dos valores supremos praticamente não funciona. Por definição, os valores supremos de uma pessoa são um ou dois. Nos estudos realizados no mundo, os valores supremos, quer dizer aqueles que recebem o número sete da escala, são tão numerosos que Schwartz estabeleceu como critério que aqueles questionários nos quais o número 7 é utilizado em mais de 21 itens devem ser eliminados e considerados inválidos (Schwartz, 2005). Desta forma, o numero 7 da escala certamente não avalia os valores supremos. Eu tenho sugerido várias estratégias para solucionar este impasse e, possivelmente, outros pesquisadores têm reagido de forma similar. Como o SVS tem sido já utilizado em tantos países e em numerosas pesquisas, Schwartz não quer introduzir mudanças nele para não prejudicar a comparação dos resultados ao longo do tempo.

O SVS foi traduzido para 46 línguas. A minha primeira contribuição nesta área foi a tradução e validação do instrumento para o português do Brasil (Tamayo \& Schwartz, 1993). Como esta atividade incluía também a possibilidade de agregar valores característicos do país para o qual o SVS estava sendo validado, organizei uma forma de identificar estes possíveis valores. Utilizando um dos métodos indicados por Rokeach (1979) para identificar valores de uma sociedade, realizei entrevistas individuais com 20 professores de escola, sacerdotes e ministros religiosos. Por meio destas entrevistas foram identificados quatro valores que parecem ser característicos da cultura brasileira (Tamayo, 1994; Tamayo \& Schwartz, 1993). Estes valores foram agregados ao SVS antes de proceder à sua validação. Desta forma, o instrumento utilizado no Brasil compreende valores transculturais e valores brasileiros, organizados em 10 tipos motivacionais. As duas publicações citadas neste parágrafo foram responsáveis pela introdução da teoria de Schwartz no Brasil e progressivamente deram origem a uma multiplicidade de pesquisas, primeiramente na UnB e, posteriormente, em outras instituições de ensino superior. Neste sentido elas têm um significado histórico. Além disso, a publicação de 1993 apresenta os resultados relativos à adequação entre a realidade observada (os valores dos sujeitos das duas amostras utilizadas) e o modelo teórico proposto (os tipos motivacionais de valores), adequação que garante a validade do SVS. Como em numerosas pesquisas realizadas posteriormente com este instrumento a estrutura dos valores tem sido repetidamente verificada, o artigo de 1993 perde parte da sua importância. Este continua sendo o ponto de referência para a validação do instrumento, mas não o único. Os resultados obtidos nas re-verificações da estrutura motivacional superam freqüentemente as divergências observadas nas duas amostras utilizadas para a validação original. Finalmente, o artigo de 1994, re-impresso neste número da revista, apresenta basicamente três tipos de resultados: a hierarquia dos valores com uma amostra brasileira, a hierarquia dos tipos motivacionais de valores e o procedimento utilizado para a identificação de valores característicos da cultura brasileira, bem como os resultados obtidos. Este último ponto é o aspecto mais original do artigo de 1994. Ao meu conhecimento, não existe na literatura brasileira sobre os valores nenhuma pesquisa ou tentativa anterior destinada a identificar, de forma empírica, valores característicos da cultura brasileira. Acho que esta foi e continua sendo uma contribuição válida. Considero, porém que, após mais de uma década da realização desta pesquisa, já seja conveniente verificar se os valores identificados continuam sendo válidos como elementos característicos da cultura brasileira e se eles se integram na estrutura motivacional dos valores da mesma forma e com a mesma intensidade observadas em 1994. Além disso, seria interessante realizar esta nova verificação com uma amostra de professores, sacerdotes e ministros religiosos mais representativa da realidade social brasileira.

Schwartz, Melech, Lehmann, Burgess e Harris (2001) e Schwartz, Lehmann e Roccas (1999) construíram um segundo instrumento para avaliação dos valores pessoais, $\mathrm{o}$ Portrait Value Questionnaire (PVQ), composto por curtos perfis ou descrições de 40 pessoas. Cada item descreve metas ou desejos que apontam para a importância dada pela pessoa a um tipo motivacional determinado. O PVQ é menos abstrato que o SVS e, em consequiência, mais adaptado para crianças e adolescentes e para pessoas com baixo nível de escolaridade. Estas características do PVQ mostram o seu 
potencial para realização de pesquisa no Brasil. Este instrumento também foi traduzido e validado por mim e os meus colaboradores para uso no Brasil, denominado Questionário de Perfis de Valores Pessoais (QPVP). Os resultados da sua validação ainda não foram publicados, mas o QPVP já tem sido utilizado em várias pesquisas em diversos lugares do país (Porto, 2005).

Rokeach (1973, p. 122) afirmava que "o conhecimento dos valores de uma pessoa nos deveria permitir predizer como ela se comportará em situações experimentais e em situações da vida real'. O estudo da relação dos valores pessoais com atitudes e comportamento tem sido um dos elementos significativos da minha linha de pesquisa. Tenho estudado a relação dos valores particularmente com quatro categorias de comportamentos e com várias reações dos trabalhadores significativas na vida organizacional: comportamento social ou geral, comportamento do consumidor, comportamento econômico, comportamento organizacional. Na primeira categoria de comportamentos tenho pesquisado, em co-autoria com vários dos meus colaboradores, por exemplo, desde a relação dos valores com comportamentos bem pontuais como o uso de camisinha no relacionamento sexual (Tamayo, Lima, Marques \& Martins, 2001) até a sua relação com o consumo de drogas (Tamayo, Nicaretta, Guimarães \& Barbosa, 1995) passando pelo comportamento ecológico (Oliveira, Trocoli \& Tamayo, 2002), a escolha de profissão (Tamayo, de Aria, Filho, Carvalho \& Bertolinni, 1998; Tamayo \& Mendonça, 2001) e a orientação política (Tamayo, Pimenta, Rolim, Rodovalho \& Castro, 1996). Em relação à categoria de comportamento do consumidor, considero que as minhas pesquisas e dos meus alunos de graduação e pós-graduação foram pioneiras nesta área, já que, a meu conhecimento, não existiam investigações no país estudando o impacto dos valores pessoais sobre este tipo de comportamento. Por outra parte, a nível internacional, a teoria dos valores de Schwartz tem sido pouco utilizada para o estudo da relação dos valores com o comportamento do consumidor. Alguns estudos publicados nesta área têm utilizado formas reduzidas ou fragmentárias do SVS sem considerar o impacto de todos os tipos motivacionais de valores sobre o comportamento do consumidor. Iniciei a minha pesquisa nesta área no ano 2000 com vários projetos que relacionavam os valores com diversos comportamentos do consumidor, como consumo de cerveja (Tamayo \& Paschoal, 2003b), intenção de compra de automóvel (Nepomuceno \& Tamayo, 2003) e compra de carro, jeans e refrigerantes (Jeunon, 2004). Foram igualmente pioneiras as pesquisas relacionando os valores pessoais com o comportamento econômico, pesquisas iniciadas na UnB sob minha orientação e mais tarde continuadas na Universidade Federal do Pará (Leite, 2003; Moreira, 1998; Moreira \& Tamayo, 1999). Finalmente, o meu interesse no estudo da relação dos valores pessoais com o comportamento nas organizações decorre da minha convicção de que o trabalho tem que estar articulado com as metas do trabalhador. Os valores pessoais são considerados como metas motivacionais que expressam alvos que a pessoa quer atingir na sua vida. Os valores, portanto, têm um papel dominante no comportamento do trabalhador e nas suas atitudes e reações no trabalho (Tamayo \& Paschoal, 2003a). Neste sentido realizei várias pesquisas junto com alguns dos meus alunos nesta direção.
Pesquisei, por exemplo, o valor preditivo dos valores em relação a comportamentos positivos e a comportamentos negativos. Como exemplos da primeira categoria podem ser mencionados os estudos relacionando os valores com o comprometimento afetivo organizacional (Tamayo, 2005) e com a cidadania organizacional (Tamayo, Moniz \& col., 1998; Porto \& Tamayo, 2005, 2002). Na segunda categoria foi estudado o poder preditivo dos valores do comportamento de retaliação nas organizações (Mendonça \& Tamayo, 2005). A relação entre valores pessoais e comportamento organizacional foi objeto também de dissertações e teses em diversas instituições do país (Matsushita, 2004; Mello, 2004). Atitudes e reações altamente relevantes na vida organizacional, tais como satisfação no trabalho (Tamayo, 2000), estresse ocupacional (Tamayo, 2001) e exaustão emocional no trabalho (Tamayo, 2002) também foram relacionadas com os valores pessoais e os resultados revelam o papel importante que estes possuem na sua incidência, desenvolvimento ou intensidade.

A minha contribuição ao estudo dos valores pessoais situa-se em níveis diferentes. Primeiramente, ao nível da medida, isto é, dos instrumentos para avaliação dos valores. Os dois instrumentos internacionalmente validados, o SVS e o PVQ foram por mim traduzidos, adaptados e validados para uso no Brasil. Eles têm sido utilizados em numerosas pesquisas e continuam sendo a base para a investigação dos valores no país. Em segundo lugar, foram por mim identificados quatro valores característicos da sociedade brasileira. Estes valores foram integrados no SVS (ou, como é conhecido no Brasil, IVS - Inventário de Valores de Schwartz) e, em conseqüência, são avaliados em toda pesquisa que utiliza este instrumento de medida. Desta forma, temos informações atualizadas sobre o grau de prioridade que estes valores possuem para os mais variados grupos sociais e profissionais. A terceira contribuição refere-se ao estudo sistemático da relação entre valores e comportamento. Esta relação é crucial para a possibilidade de aplicação prática da teoria dos valores e dos resultados obtidos nas pesquisas. A associação dos valores com o comportamento tem sido verificada inúmeras vezes no país e com os mais variados comportamentos. Várias pesquisas atuais têm como critério a aplicabilidade dos seus resultados. Isto explica talvez a importância dada nos estudos da relação entre valores e determinadas categorias de comportamento tais como organizacional, ecológico e do consumidor. Finalmente, cabe salientar que, graças às pesquisas realizadas nesta área, já possuímos no país um acervo importante de conhecimentos relativos ao poder preditivo dos valores pessoais sobre diversos comportamentos. Não é a minha intenção sintetizar esses resultados neste texto, mas eles estão disponíveis nas numerosas publicações, dissertações e teses realizadas nesta área.

\section{Valores laborais}

Os valores pessoais ou valores básicos estão estruturados em duas categorias. A primeira refere-se a uma estrutura geral e integra os valores relacionados com todos os aspectos da vida. A segunda compreende estruturas de valores associados a contextos específicos da vida, tais como o sexo, a religião, a família, o trabalho. Segundo Schwartz (1992), a avaliação de valores específicos não tem como objetivo revelar os valores 
básicos da pessoa, mas clarificar diferenças interpessoais e interculturais que podem surgir quando valores são expressos em julgamentos e comportamentos específicos. Na área de valores relacionados a situações específicas, pesquisei os valores sexuais (Tamayo, 1993; Tamayo \& Barbosa, 1993; Tamayo, Resende, Barros, Madureira \& Souza, 1995) e os valores relativos ao trabalho. Dentre os contextos específicos, o contexto do trabalho é aquele que mais freqüentemente tem sido objeto de estudo, particularmente nos últimos anos. Ao analisar a literatura nesta área encontram-se dois problemas críticos: a escassez de instrumentos de medida e, particularmente, a ausência de modelos teóricos para os mesmos. Assim, a minha primeira preocupação nesta área foi com a construção de escalas apropriadas para a avaliação dos valores relativos ao trabalho. Dois instrumentos de medida foram desenvolvidos no contexto de duas teses de doutorado por mim orientadas: o IST, Inventário de Significado do Trabalho (Borges, 1996; 1999) e a EVT, Escala de Valores relativos ao Trabalho (Porto \& Tamayo, 2003); o primeiro validado com pessoas analfabetas e, o segundo, com trabalhadores que possuíam pelo menos $2^{\circ}$ grau completo. Com este segundo instrumento, várias pesquisas têm sido realizadas (Barrella, 2003; Lopes, 2004; Paschoal \& Tamayo, 2005; Rodrigues, 2005;) e outras estão em andamento tanto na área da psicologia quanto da administração.

Duas contribuições podem ser destacadas nesta área: o modelo utilizado e os instrumentos em si. 1) O conceito de modelo é fundamental na ciência. $\mathrm{O}$ modelo consiste numa representação simplificada da realidade. O modelo utilizado para os dois instrumentos tem o mérito de inserir os valores laborais no contexto da teoria dos valores humanos, já que aqueles se referem a um contexto específico da vida das pessoas. Portanto, as duas dimensões bipolares observadas nos valores pessoais (abertura a mudança versus conservação e autotranscendência versus autopromoção) foram utilizadas para a elaboração do modelo. Supõe-se, portanto, que na realidade os valores laborais se organizam em torno a estes quatro pólos ou fatores que expressam a síntese das motivações subjacentes aos valores humanos. No caso do IST, o modelo foi explicativo, quer dizer a posteriori, o modelo foi utilizado para explicar os fatores obtidos na sua validação de forma a integrá-los na teoria geral dos valores. Já no caso da EVT, o modelo precedeu a sua construção constituindo uma representação da forma como se estruturam os valores laborais. Assim, tanto o IST quanto a EVT, relacionam-se com as dimensões de ordem superior da teoria dos valores pessoais. 2) Os dois instrumentos construídos e validados constituem uma contribuição importante visto que não existia no país nenhum instrumento validado para a avaliação dos valores relativos ao trabalho. A pesquisa nesta área estava inviabilizada por falta de instrumentos de medida validados. Atualmente, os dois instrumentos estão contribuindo para o desenvolvimento da pesquisa relativa aos valores laborais.

\section{Valores organizacionais}

Os valores organizacionais constituem um conjunto dentro do universo dos valores. Eles referem-se ao domínio específico das organizações. A organização escolhe os seus valores desde os alvores da sua existência, desde o momento mesmo da sua fundação. Freqüentemente, os valores precedem a existência mesma da organização. A razão é simples, já que, como afirma Nietzsche, "os valores caracterizam a vontade de existir e de se afirmar de uma coletividade". Assim, por meio dos valores a organização expressa as suas metas e se afirma como diferente na sociedade e no mercado. Para a escolha dos seus valores e da prioridade dada aos mesmos, a organização inspira-se nos valores da sociedade e nos valores dos seus membros, particularmente dos fundadores e daqueles com maior poder de influência. A minha contribuição nesta área começou na área da medida já que não existia no país nenhum instrumento validado para avaliação dos valores organizacionais. Três escalas foram construídas e validadas utilizando nelas a percepção dos trabalhadores para avaliar os valores da organização: A Escala de Valores Organizacionais (Tamayo \& Gondim, 1996), o Inventario de Valores Organizacionais (Tamayo, Mendes \& Paz, 2000) e o Inventário de Perfis de Valores Organizacionais (Oliveira \& Tamayo, 2004). A primeira foi construída sem um modelo explícito, mas de forma empírica. De fato, na mente dos pesquisadores estava presente um modelo que era o dos valores culturais de Schwartz, mas ele não foi utilizado para a construção dos itens. O modelo cultural de Schwartz postula três dimensões bipolares: autonomia versus conservadorismo, hierarquia versus igualitarismo e domínio versus harmonia. A idéia era verificar se por meio de um levantamento empírico, em que os participantes apresentassem valores da sua respectiva organização, se podiam identificar itens para os seis fatores propostos no modelo cultural de Schwartz. Assim, a base para a construção dos itens foi um levantamento realizado com empregados de cinco organizações diferentes, públicas e privadas. O questionário solicitava aos sujeitos que relacionassem, no espaço previsto, cinco valores da sua organização. Desta forma, obteve-se uma lista de mais de 500 valores que após análise de conteúdo e eliminação dos valores sinônimos ficou reduzida a 48 valores. Para os fatores autonomia e igualitarismo foram encontrados pouquíssimos itens. Este resultado foi interpretado como ausência relativa dos valores de igualitarismo e autonomia em empresas brasileiras. A Escala de Valores Organizacionais, portanto, foi construída a partir dos 48 valores identificados no levantamento empírico. Apesar da falta de um referencial teórico para a sua construção, esta escala tem sido utilizada em várias pesquisas.

O segundo instrumento de medida construído foi o Inventário de Valores Organizacionais e tinha como objetivo superar a deficiência apontada no instrumento anteriormente descrito. O Inventário de Valores Organizacionais integra duas abordagens diferentes: a empírica e a teórica. Os itens que haviam sido levantados empiricamente por Tamayo e Gondim (1996) para a construção da Escala de Valores Organizacionais foram utilizados para este novo instrumento, já que eles expressavam adequadamente vários dos construtos axiológicos que se pretendia medir, de acordo com o modelo dos valores culturais de Schwartz. Para representar de forma apropriada os tipos motivacionais de valores que não foram suficientemente representados na escala de Tamayo e Gondim, foram construídos novos itens. Estes foram construídos a partir de pressupostos teóricos, procurando valores que expressassem adequadamente os pólos de igualitarismo 


\section{A. Tamayo}

e autonomia. A análise multidmensional confirmou os seis fatores esperados. Assim, os pressupostos teóricos foram confirmados, já que as três dimensões propostas foram encontradas.

O terceiro instrumento, o Inventario de Perfis de Valores Organizacionais (IPVO), foi construído no contexto de uma tese de doutorado (Oliveira, 2004). Para este instrumento utilizamos um modelo diferente que eu nunca tinha visto ser utilizado para construção de um instrumento de valores organizacionais. Não encontrei nenhuma referência neste sentido na literatura. $\mathrm{O}$ esperado é utilizar o modelo dos valores culturais já que cada organização é composta por um grupo de pessoas com normas e objetivos próprios que pode ser considerado como uma cultura específica. Para a construção do IPVO utilizei o modelo dos valores pessoais composto por 10 tipos motivacionais de valores. Isso significa postular paralelismo entre os valores pessoais e os valores da organização. Esse é um problema que não parece ter sido estudado empiricamente. Eu não encontrei nenhuma publicação sobre esta temática. Contudo, para a compreensão da adaptação do trabalhador ao seu ambiente de trabalho, é fundamental investigar a possibilidade de paralelismo entre os valores pessoais e os valores organizacionais. $\mathrm{O}$ paralelismo axiológico postulado para a construção do IPVO consiste na correspondência biunívoca dos tipos motivacionais de valores do trabalhador e dos valores organizacionais, isto é, relação entre cada tipo motivacional de valores do trabalhador com apenas um fator de valores da organização. Isso implica em correspondência de metas entre o trabalhador e a organização. Por falta de espaço não discuto neste texto as bases teóricas para este pressuposto (ver Tamayo, no prelo). Para a construção dos itens foram formuladas definições constitutivas e operacionais dos tipos motivacionais de valores organizacionais, baseando-se na teoria dos valores pessoais. A partir dessas definições, itens foram construídos para cada um dos tipos de valores. A validação foi realizada por meio de análise fatorial (Oliveira \& Tamayo, 2004).

Com os três instrumentos para avaliação dos valores organizacionais acima descritos várias pesquisas, dissertações e teses foram realizadas e outras estão em andamento tanto na psicologia quanto na administração em diversos lugares do país (Latorre, 2006; Louback, 2006; Miguel, 2005; Pereira, 2003; Pupak, 2003; Rossoi, 2002). As principais pesquisas realizadas por mim ou em co-autoria com outros pesquisadores estudaram a relação dos valores organizacionais com estresse ocupacional (Tamayo, no prelo), comprometimento afetivo organizacional (Tamayo, 2005), civismo nas organizações (Porto \& Tamayo, 2005), satisfação no trabalho (Tamayo, 1998) e prazer-sofrimento no trabalho (Mendes \& Tamayo, 2001).

Como sintetizar a minha contribuição para a compreensão e o estudo dos valores organizacionais? As contribuições mais significativas situam-se em cinco níveis diferentes: importância dada aos valores organizacionais; estratégias de avaliação dos valores organizacionais; modelos teóricos para a sua avaliação; instrumentos construídos e validados e conjunto de resultados obtidos nas pesquisas.

1) A primeira contribuição situa-se, sem dúvida, na própria escolha de estudar os valores organizacionais. Segundo Rokeach (1979), poucos pesquisadores têm-se interessado por este tipo de estudo. ("With only a few exceptions investigators have not concerned themselves with values and the organization", p. 71). Apesar desta afirmação de Rokeach ter já quase 30 anos, ela ainda reflete certo desinteresse dos pesquisadores pelo estudo dos valores organizacionais. É sintomático que a teoria de Schwartz, apesar do seu enorme poder heurístico, praticamente não tenha sido utilizada para o estudo dos valores das organizações. Considero, portanto, que a simples opção pelo estudo sistemático e empírico dos valores organizacionais constitui uma contribuição válida para o desenvolvimento desta área.

2) Estratégias de avaliação dos valores organizacionais. Três estratégias de identificação e mensuração dos valores organizacionais encontram-se descritas na literatura. A primeira consiste em identificar os valores da organização a partir dos valores individuais dos seus membros, a segunda avalia os valores a partir de documentos oficiais da empresa (relatórios anuais, estatutos, discursos, revistas internas...), a terceira abordagem consiste em utilizar a percepção dos trabalhadores para avaliar os valores da organização. Esta última abordagem tem sido desenvolvida particularmente no Brasil (Oliveira \& Tamayo, 2004; Tamayo, 1996; Tamayo \& cols., 2000; Tamayo \& Gondim, 1996), A minha contribuição e a dos meus colegas nesta área tem sido significativa. Esta abordagem estuda os valores organizacionais e a sua hierarquia de acordo com a representação mental que os empregados têm do sistema axiológico da empresa. Para fazer este tipo de estudo utilizam-se questionários previamente elaborados a partir de várias fontes: análise de documentos de empresas, entrevistas com empregados de várias organizações e modelos teóricos existentes na literatura. Os valores da empresa e o seu conteúdo não são definidos a priori, mas a partir da realidade da empresa e do que já existe na literatura. Esta abordagem adota, portanto, uma perspectiva de integração das informações provenientes da empresa e da literatura científica. Além disso, os valores apresentados no questionário são avaliados pelos trabalhadores por meio de uma escala de intensidade, de forma a expressar mais diretamente a prioridade percebida para cada um dos valores analisados. Este método permite identificar a importância acordada a cada um dos valores e determinar o grau ou a intensidade de compartilhamento dos valores entre os membros da organização.

3) Dois modelos teóricos diferentes foram elaborados para o estudo dos valores organizacionais: o modelo dos valores culturais de Schwartz e o modelo dos valores pessoais. O primeiro considera a organização como uma cultura e o segundo como um empreendimento definido por metas grupais e pessoais.

4) Os três instrumentos de medida construídos e validados para a avaliação dos valores organizacionais constituem, sem dúvida, uma valiosa contribuição para a realização de pesquisa nesta área. Convém salientar que não existia no país nenhum instrumento validado para avaliação dos valores organizacionais e que, talvez por causa desta deficiência, a pesquisa nesta área era inexistente.

5) Contribuição teórica. As conclusões dos estudos realizados por mim e pelos meus colaboradores constituem um conjunto de conhecimentos inéditos que, além de contribuir para a compreensão da vida organizacional, apresentam grande aplicabilidade na gestão das organizações. Trata- 
se, portanto, de uma contribuição significativa ao nível da teoria. Isto é valido particularmente na área da influência dos valores organizacionais sobre o estresse ocupacional, o comprometimento organizacional, a cidadania, a satisfação no trabalho e o prazer-sofrimento no trabalho. Muitos outros aspectos teóricos, igualmente significativos, têm sido desenvolvidos por meio das numerosas pesquisas realizadas por colegas de outras instituições de ensino superior, tanto na psicologia quanto na administração, nos mais variados lugares do Brasil.

\section{Conclusão}

Nas páginas precedentes discuti, brevemente, alguns elementos bastante objetivos que podem ser considerados como indicadores da minha contribuição ao estudo dos valores pessoais, laborais e organizacionais. Sem dúvida, eles são indicadores válidos. Contudo, considero que o indicador mais importante é a quantidade e a qualidade de pessoas que estão atualmente realizando pesquisa na área dos valores no Brasil. Atualmente existem vários núcleos ou centros de pesquisa sobre os valores. Nesses, bem como em diversas outras universidades, existe um número significativo de dissertações de mestrado e teses de doutorado que estão sendo elaboradas. Considero, portanto, que a semente lançada no início dos anos 1990 frutificou e atualmente existe no Brasil uma frondosa floresta de pesquisas axiológicas.

\section{Referências}

Anderson, N. H. (1968). Likableness ratings of 555 personalitytrait words. Journal of Personality and Social Psychology, 9, 272-279.

Barrella, F. A. (2003). Competências gerenciais: Um estudo sobre a influencia de valores relativos ao trabalho de gestores. Dissertação de Mestrado. Universidade Presbiteriana Mackenzie, São Paulo.

Borges, L. (1996). Significado do trabalho e socialização Tese de Doutorado. Universidade de Brasília, Brasília.

Borges, L. (1999). A estrutura fatorial dos atributos valorativos descritivos do trabalho: Um estudo empírico de aperfeiçoamento e validação de um questionário. Estudos de Psicologia, 4, 107-139.

Günther, H. (1981). Uma tentativa de traduzir e adaptar a escala de valores de Rokeach para uso no Brasil. Arquivos Brasileiros de Psicologia, 33(3), 58-72.

Jeunon, E. E. (2004). Prioridades axiológicas e orientação de consumo: validação de um modelo integrativo. Tese de Doutorado, Universidade de Brasília, Brasília.

Latorre, S. Z. (2006). Perfis de cultura e de valores organizacionais: Um estudo em empresas de tecnologia. Dissertação de Mestrado, Universidade Presbiteriana Mackenzie, São Paulo.

Leite, I. D. L. (2003). Expectativas quanto ao primeiro emprego: estrutura conceitual e relação com valores. Dissertação de Mestrado, Universidade Federal do Pará, Belém.

Lopes, T. A. R. (2004). Valores do trabalho e valores organizacionais: Um estudo multicaso. Dissertação de Mestrado, Universidade Presbiteriana Mackenzie, São Paulo.
Louback, J. (2006). Valores organizacionais e racionalidades: Uma visita ao Terceiro Setor. Dissertação de Mestrado, Universidade Presbiteriana Mackenzie, São Paulo.

Matsushita, M. (2004). A influencia dos valores dos gestores no papel de recursos humanos. Dissertação de Mestrado, Universidade Presbiteriana Mackenzie, São Paulo.

Mello, N. O. (2004). A influencia dos valores dos gestores na orientação para relações de confiança. Dissertação de Mestrado, Universidade Presbiteriana Mackenzie, São Paulo.

Mendes, A. M. \& Tamayo, A. (2001). Valores organizacionais e prazer-sofrimento no trabalho. PsicoUSF, 6(1), 39-46.

Mendonça, H. \& Tamayo, A. (2005). Valores e retaliação organizacional. Em A. Tamayo \& J. B. Porto (Orgs.), Valores e comportamento nas organizações (pp. 145-159). Petrópolis: Vozes.

Miguel, L. A. P. (2005). Os valores organizacionais e a criação do conhecimento. Dissertação de Mestrado, Universidade Presbiteriana Mackenzie, São Paulo.

Moreira, A. (1998). Valores e dinheiros: um estudo transcultural das relações entre prioridades de valores e significado do dinheiro para indivíduos. Tese de Doutorado, Universidade de Brasília, Brasília.

Moreira, A. \& Tamayo, A. (1999). Escala de significado do dinheiro: desenvolvimento e validação. Psicologia: Teoria e Pesquisa, 15(2), 93-105.

Nepomuceno, M. V. \& Tamayo A. (2003). Valores pessoais e intenção de compra de automóvel. [Resumo]. Em Sociedade Brasileira de Psicologia (Org.), Resumos de comunicações cientificas (pp. 69-70). Belo Horizonte: SBP.

Oliveira, A. F. (2004). Confiança do empregado na organização: impacto dos valores pessoais, organizacionais e da justiça organizacional. Tese de Doutorado, Universidade de Brasília, Brasília.

Oliveira, A. F. \& Tamayo, A. (2004). Inventario de Perfis de Valores Organizacionais. Revista de Administração (USP), 39, 129-149.

Oliveira, C. P., Trocoli, B. \& Tamayo, A. (2002). Values and ecological behavior: An empirical study. Em Association for people environemal studies (Org.), La Coruña culture, quality of life and globalization. Problems and challengens for the new millennium (p. 263). La Coruña: APES.

Paschoal, T. \& Tamayo, A (2005). Impacto dos valores laborais e da interferência família-trabalho no estresse ocupacional. Psicologia: Teoria e Pesquisa, 21(2), 173-180.

Pereira, J. A. S. (2003). Vivencias de prazer-sofrimento na atividade gerencial em empresa estratégica: o impacto dos valores organizacionais. Dissertação de Mestrado, Universidade de Brasília, Brasília.

Porto, J. B. (2005). Mensuração de valores no Brasil. Em A. Tamayo \& J. B. Porto (Orgs.) Valores e comportamento nas organizações (pp. 96-119). Petrópolis: Vozes.

Porto, J. B. \& Tamayo, A. (2002). Prioridades axiológicas e regiões brasileiras: Preditores de civismo nas organizações. Psicologia Organizações e Trabalho, 2(1), 31-61.

Porto, J. B. \& Tamayo, A. (2003). Escala de Valores relativos ao Trabalho - EVT. Psicologia: Teoria e Pesquisa, 19(2), 145152.

Porto, J. B. \& Tamayo, A. (2005). Valores organizacionais e civismo nas organizações. Revista de Administração Contemporânea $-R A, 9(1), 35-52$. 


\section{A. Tamayo}

Pupak, M. O. (2003). Identificação dos valores organizacionais do Instituto de Pesquisas Energéticas e Nucleares-IPEN. Tese de Doutorado, Universidade de São Paulo, São Paulo.

Rodrigues, M. (2005). Hierarquia de valores do trabalho: A influencia do assedio moral. Dissertação de Mestrado, Universidade Presbiteriana Mackenzie, São Paulo.

Rokeach, M. (1973). The nature of human values. New York: The Free Press.

Rokeach, M. (1979). Understanding human valures New York: Free Press.

Rossoi, E. Z. (2002). A cultura e os valores organizacionais de uma empresa financeira estatal e as (não)decisões dos seus gerentes. Dissertação de Mestrado, Universidade Católica de Goiás, Goiânia.

Schwartz, S. H. (1992). Universals in the content and structure of values: theoretical advances and empirical tests in 20 countries. Em Zanna, M. (Org.), Advances in Experimental Social Psychology (pp 1-65). Orlando: Academic.

Schwartz, S. H. (2005). Valores humanos básicos: seu contexto e estrutura intercultural. Em A. Tamayo \& J. Porto (Orgs.), Valores e comportamento nas organizações (pp. 21-55). Petrópolis: Vozes.

Schwartz, S. H. \& Bilsky, W. (1987). Toward a Universal Psychological Structure of human values. Journal of Personality and Social Psychology, 53, 550-562.

Schwartz, S. H., Lehmann, A. \& Roccas, S. (1999). Multimethod probes of basic human values. Em J. Adamopoulos \& Y. Kashima (Orgs.), Social psychology and culture context: Essays in honor of Harry C. Triandis (pp. 107-123). Newbury Park: Sage.

Schwartz, S. H., Melech, G., Lehmann, A., Burgess, S. \& Harris, M. (2001). Extending the cross-cultural validity of the theory of basic human values with a different method of measurement. Journal of Cross-cultural Psychology, 32, 519-542.

Tamayo, A. (1988). Influência do sexo e da idade sobre o sistema de valores. Arquivos Brasileiros de Psicologia, 38(3), 91-104.

Tamayo, A. (1993). Escala de valores sexuais. Em Sociedade Brasileira de Psicologia (Org.) Resumos de comunicações cientificas (p. 102). Ribeirão Preto: SBP.

Tamayo, A. (1994). Hierarquia de valores transculturais e brasileiros. Psicología: Teoria e Pesquisa, 10, 269-285.

Tamayo, A. (1998). Valores organizacionais: sua relação com satisfação no trabalho, cidadania organizacional e comprometimento afetivo. Revista de Administração (USP), 33(3), 56-63.

Tamayo, A. (2000). Prioridades axiológicas e satisfação no trabalho. Revista de Administração (USP), 35(2), 37-47.

Tamayo, A. (2001). Prioridades axiológicas, atividade física e estresse ocupacional. Revista de Administração Contemporânea $-R A C, 5,127-147$.

Tamayo, A. (2002). Exaustão emocional no trabalho. Revista de Administração (USP), 37(2), 26-37.
Tamayo, A. (2005). Impacto dos valores pessoais e organizacionais sobre o comprometimento organizacional. Em A. Tamayo \& J. Porto (Orgs.), Valores e comportamento nas organizações (pp. 160-186). Petrópolis: Vozes.

Tamayo, A. (no prelo). Valores organizacionais e estresse no trabalho. Revista de Administração Contemporânea-RAC.

Tamayo, A. \& Barbosa, V. M. (1993). Valores sexuais de adolescentes de escolas publica e privada. Em Sociedade Brasileira de Psicologia (Org.), Resumos de comunicações cientificas (p. 104). Ribeirão Preto: SBP.

Tamayo, A., de Aria, J. B., Filho, A. B., Carvalho, E. M. \& Bertolinni, V. (1998). Diferenças nas prioridades axiológicas de músicos e advogados. Psicologia: Reflexão e Crítica, 11(2), 281-293.

Tamayo, A. \& Gondim, M. G. C. (1996). Escala de valores organizacionais. Revista de Administração (USP), 31(2), 6272.

Tamayo, A., Lima, A. Marques, J. \& Martins, L. (2001). Prioridades axiológicas e uso de preservativo. Psicologia: Reflexão $e$ Crítica, 14(1), 151-159.

Tamayo, A., Mendes, A. M. \& Paz, M. G. T. (2000). Inventario de valores organizacionais. Estudos de Psicologia (Natal), 5(2), 289-315.

Tamayo, A. \& Mendonça, H. (2001). Prioridades axiológicas e escolhas acadêmico-profissionais: dados empíricos e perspectivas teóricas. Estudos Vida e Saúde, 28(4), 625-638.

Tamayo, A., Moniz, A. L. F., de Oliveira, V. B., Carvalho, R. S., Armando, P. \& Guimarães, F. T. (1998). Prioridades axiológicas, tempo de serviço e cidadania organizacional. Psicologia: Teoria e Pesquisa, 14(1), 45-50.

Tamayo, A., Nicaretta, M., Ribeiro, R. \& Barbosa, L. d. P. G. (1995). Prioridades axiológicas y consumo de drogas. Acta Psiquiátrica y Psicológica de América Latina, 31, 300-307.

Tamayo, A. \& Paschoal, T. (2003a). A relação da motivação para o trabalho com as metas do trabalhador. Revista de Administração contemporânea $R A C, 7,33-54$.

Tamayo, A. \& Paschoal, T. (2003b). Valores como predictores de consumo de cerveza. [Resumo]. Em Sociedad Interamericana de Psicologia (Org.), Resúmenes (p. 52). Lima: SIP.

Tamayo, A., Pimenta, M. L., Rolim, M. M. A., Rodovalho, O. R. M. \& Castro, P. M. R. (1996). Prioridades axiológicas e orientação política. Psicologia: Teoria e Pesquisa, 12(3), 253-259.

Tamayo, A., Resende, T. I. M., Barros, A. L., Madureira, A. F. A. \& Souza, J. C. (1995). Valores sexuais, gênero e pratica religiosa. Em Sociedade Brasileira de Psicologia (Org.), Resumos de comunicações cientificas (p. 370). Ribeirão Preto: SBP.

Tamayo, A. \& Schwartz, S. H. (1993). Estrutura motivacional dos valores humanos. Psicología: Teoria e Pesquisa, 9(2), 329-348.

Wach, M. \& Hammer, B. (2003). La structure des valeurs est-elle universelle? Genèse et validation du modèle compréhensif de Schwartz. Paris: L'Harmattan. 